\title{
TEXTSORTEN - NATÜRLICHE SPRACHFORMEN ODER KULTURELLE SPRACHFORMEN?
}

\section{Etablierung der Textsortenlehre in der Textlinguistik}

Hinsichtlich dieser Problematik befinden wir uns in der textlinguistischen Begriffsbildung. Textsorte ist ein Begriff der Textlinguistik, der seit der Wende von den $60 \mathrm{er}$ zu den 70er Jahren etablierten Disziplin der Sprachwissenschaft. Diese galt damals als neu. ${ }^{1}$

Beim zweiten Teil der Überschrift läßt sich nicht davon absehen, daß darin der Rückbezug auf den frühen Titel André Jolles $\left({ }^{1} 1930\right)$ : Einfache Formen wirksam ist. Man kann bei Jolles eine frühe reflektierte Kategorisierung von Texten beobachten. Zwischen dem linguistischen Abstraktum Text überhaupt, dem Terminus für diese hochkomplexe verbale Erscheinung (Textbegriff), und dem konkreten Einzeltext, den man eigentlich erlebt (realisierter Text), wird eine weitere Abstraktions- bzw. Begriffsebene mit der Textkategorie Textsorte (Textsortenbegriff) eingezogen. Die Form (vom Text) bei Jolles entspricht der Textsorte in anderen Textaufkommen. Die einfache Form Witz z.B. ist das Paradigma für alle einzelnen Witze. Hier läßt sich auch gut die Koinzidenz zwischen Jolles'scher Textform und der später sogenannten Textsorte zeigen, denn fast ein halbes Jahrhundert später erleben wir die terminologische Metamorphose in der Studie Bernhard Marfurt (1977): Textsorte Witz.

Zwar wurden bei Jolles aufgeführte Formen, so Märchen und Sage, schon bei Jacob und Wilhelm Grimm zusammengefaßt, jedoch unter einem anderen Interesse. Gibt Jolles eine literaturwissenschaftliche Betrachtung über textliche Erscheinung und Wirkung dieser sprachlichen Formen, so ist es den Grimms primär um die Dokumenta-

1 Vgl. Titel damaliger Beiträge: P. Hartmann (1968), Textlinguistik als neue linguistische Teildisziplin; K. Brinker (1971), Aufgaben und Methoden der Textlinguistik. Kritischer Überblick über den Forschungsstand einer neuen linguistischen Teildisziplin; K. Brinker (1972), Textlinguistik. Zum Forschungsstand einer neueren linguistischen Teildisziplin.

An der von W. Dressler (1978) herausgegebenen Sammlung wird mit Aufsätzen von 1912 bis 1972 die Forschungsgeschichte einer Textlinguistik vor der Orientierung auf Textsorten bzw. Texttypik, Texttypologie gezeigt.

Unter der Basisliteratur für das germanistische Studium werden bei W. Kürschner (1994: 87f.) Titel als Lehrbücher für die Textlinguistik genannt. 
tion der Belege für das volksgeistige Schaffen zu tun. Für die Grimms handelt es sich sozusagen um sprachliche Naturformen.

Von dem von der Literaturwissenschaft etablierten Kanon dichterischer Texte, den diese nach Gattungen organisiert, sind die Einfachen Formen als Volkspoesie separiert und in fachwissenschaftlichem Zusammenhang der sog. Volkskunde (z.B. Hermann Bausinger, (1980): Formen der "Volkspoesie") gewürdigt worden. Das Anliegen von A. Jolles war, die literarische Dignität der von ihm beobachteten Textformen zu erweisen, auch wenn sie ihm der Gestaltung und Funktion nach als Einfache Formen galten. Er neigt dazu, sie zu den literarischen Gattungen zu rücken. Diese Aufwertung war für manche der Einfachen Formen wohl auch erfolgreich.

Übrigens ist mit Gattung ein dritter Terminus aufgetaucht, der gemeinsam mit Textsorte und (Einfache) Form in den gleichen linguistischen Begriffshorizont gehört. Jeder Einzeltext der Dichtung bzw. poetischen Literatur beansprucht die Zuordnung zu einer literarischen Gattung. Die Begriffsdifferenzierung von Textsorte und Gattung gehört schon zu den traditionellen Zugriffen der Textlinguistik. Beleg dafür ist die Aufsatzsammlung Textsortenlehre - Gattungsgeschichte (1977), Hrsg. von W. Hinck, welche den Anregungen des Germanistentages 1976 entspringt. ${ }^{2}$ Später erscheint Textsorten und literarische Gattungen (1983) als Dokumentation des Germanistentages 1979.

Ein in der Textlinguistik früher Versuch der Textklassifikation ist der von P. Kern (1969). Kern bemüht sich um die Klassifikation eines breiten Spektrums von Texten, und zwar in Texttypen, wie er sie nennt. Der Terminus Typ für die Textklasse setzte sich aber nicht durch.

$\mathrm{Zu}$ Beginn der Suche nach Prototypen oder Textklassen ist die Auffassung so, daß man die Menge der Texte auf Textsorten, Einfache Formen und Gattungen verteilt sieht. Für das System oder den Zusammenhang dieser Textklassifikation neigte man anfänglich zur Benennung Typik/Texttypik. Auch Texttypologie kommt auf, wird gar zum vorherrschenden Terminus in der Textwissenschaft. ${ }^{3}$

Die jüngste und erfolgreiche unter den Bezeichnungen für die Textklasse in der Typologie ist Textsorte; ihr Erfolg geht zu Lasten des synonymen Texttyp und hängt wohl mit dessen Vermeidung zusammen. Erinnerlich ist, daß gleichzeitig mit den Klassifikationsversuchen der Textlinguistik auch die Theologie versuchte, Zusammenhänge zwi-

In der Einführung zu dieser Aufsatzsammlung, Seite V, folgende Standortbestimmung: "Der Germanistentag 1976 hatte sich zur Aufgabe gesetzt, diese Lage kenntlich zu machen und die Notwendigkeit eines engeren Zusammenwirkens zwischen Sprach- und Literaturwissenschaft aufzuzeigen. An Schwerpunkten der gegenwärtigen Forschung sollte das neue Spektrum der deutschen Philologie vorgewiesen und dabei auch ein Begriff von der wiedergewonnenen Arbeitsenergie vermittelt werden."

3 Nachweisbar im terminologischen Lexikon und in der Bibliographie: H. Bußmann ( $\left.{ }^{2} 1990: 782\right)$;. D. Kreuder ( $\left.{ }^{3} 1993: 91\right)$. 
schen ihren Texten unter neuen Aspekten zu verstehen. Mit Hilfe der Termini Typ, Antityp, Typos, Typologie wurde ein eigenes Textverständnis konzipiert, womit diese und ähnlich klingende Termini als besetzt erschienen. Erzählungen des Alten Testamentes als Typ werden als Vorausdeutungen solcher des Neuen Testamentes verstanden. Das Beziehungssystem insgesamt ist die Typologie. Der Zusammenhang ist repräsentativ bei L.eonhard Goppelt (1939/Nachdruck 1990) dargestellt.

Die linguistische Texttypik erscheint zunächst so aufgefaßt, daß den Gattungen die Texte der Dichtung, den Einfachen Formen die Texte der Volkspoesie und den Textsorten die sonstigen Texte der alltags-, fach- und sondersprachlichen Kommunikation zugeordnet werden.

Der Erarbeitungsvorgang einer Textsortenlehre für das Deutsche läßt sich an einem linguistischen Unternehmen konkretisieren und veranschaulichen. Die Aufgabe der Textsortenklassifizierung bestand in dem von $\mathrm{H}$. Steger initiierten Vorhaben "Grundstrukturen der gesprochenen Sprache" von 1966. Dieses Vorhaben war in den Gesamtzusammenhang des Projekts "Grunddeutsch", später "Grundstrukturen der deutschen Sprache", des Instituts für deutsche Sprache/Mannheim eingeordnet. In den Publikationen aus dieser Forschungsarbeit, die in der Forschungsstelle Freiburg des IDSMannheim durchgeführt wurden, findet sich der als Typik bezeichnete Begriff für die Resultate der Klassifikation. Auf frühere Titel und Stellen sei hingewiesen:

Typik bei H. Steger (1967: 272, 276) ${ }^{4}$ H. Steger (1969: 82), P. Kern (1969: 8, 13, 19), U. Engel (1969: 81), K.-H. Bausch (1971: 9, 10, 15), P. Schröder (1973: 13, 15, 24, 25), R. Müller (1973: 67), K.-H. Deutrich (1973: 114), G. Schank/G. Schoenthal $\left({ }^{2} 1983: 4\right)$.

Typologie bei H. Steger (1967: 291 durch Nennung von H. Zimmermann (1965)), P. Kern (1969: 8), P. Schröder (1973: 14), E. Werlich ( ${ }^{2}$ 1979: 12, 56, 100, 105, 106).

Es hätte nahegelegen, die einzelne Textklasse Typ zu nennen, zumal Typik wie eine Ableitung davon erscheint. Auch von Typologie her, das gleichzeitig und immer intensiver benutzt wird, hätte sich das angeboten. Statt dessen wird aber der sehr innovativ wirkende Terminus Textsorte eingeführt. Das Prototypische ähnlicher Texte wird als Textsorte begriffen; und es gilt auch Textsorte, wenn die jeweils andere Ausprägung der Ähnlichkeit als bei Einfacher Form und Gattung vorliegt. Die Einführung von Textsorte vollzieht sich in Publikationen, die an das zuvor schon genannte Forschungsprojekt des IDS gebunden waren:

Textsorte bei P. Kern (1969: 13, 14), ${ }^{5}$ K.-H. Deutrich (1971: 27), (1971b: 40, 41, 47), R. Müller (1971: 123, 124), P. Schröder (1973: 13, 15, 26, 40, 42), R. Müller (1973: 54, 63, 67, 68, 69, 72, 73), K.-H. Deutrich (1973: 114), Deutrich, Karl-

4 Die im folgenden aufgeführten Positionen der Termini können immer durch den Vermerk et passim ergänzt werden. 
Helge/Schank, Gerd (1973: 251), H. Steger u.a. (1974: 39, 40, 41, 62, 65, 66, 69, 87, 89, 90, 91, 92, 93, 95), U. Elmauer u. R. Müller (1974: 98 bis 116), G. Schank/G. Schoenthal ( $\left.{ }^{2} 1983: 4,5,40,42\right)$.

Texttyp ist auch zu beobachten: H. Steger (1969: 82, 92, 99), P. Kern (1969: 13, 15, 20), W. Müller (1969: 71), K.-H. Deutrich (1973: 145, 146), H. Steger u.a. (1974: 39, 55, 66, 69, 87), E. Werlich $\left({ }^{2} 1979: 27 \mathrm{ff} ., 37 f ., 44,56,70,101\right)$. Textsorte findet sich bei Werlich auch, jedoch selten und mit Bezug auf andere Literatur zur Texttypologie; S. 116 wird ausdrücklich die eigene Vermeidung des Begriffs Textsorte konstatiert.

Bei P. Schröder (1973a: 26) und R. Müller (1973: 67f.) sind erste aus der Zeit der Vordiskussion stammende Definitionen für Textsorte präsentiert. Sie gehen dahin, daß eine Textsorte solche Texte umfaßt, die mit übereinstimmenden situativen Merkmalen bzw. Äußerungsbedingungen beschrieben werden, also gleichen Situationen entstammen. Die Normsituation für eine Textsorte wurde Redekonstellationstyp genannt. Den einzelnen von den einer Textsorte zugesellten Texten wurde die Benennung Textexemplar zuteil. Es war also eine Typik/Typologie gesprochener Texte entwickelt, in der z.B. das Textexemplar aus einer bestimmten Redekonstellation unter die Textsorte Interview eingeordnet wird. ${ }^{6}$

\section{Graphische Textsortendarstellung in der Matrix}

Die Textklassifikation führte zu weitgehender Differenzierung, so daß sich anbot, durch graphische Darstellung die Übersicht auf einen Blick zu gewährleisten. Die eigentliche Ausprägung und entscheidende Publizität wurde dann durch die Präsentation im Funk-Kolleg Sprache II (1973: 250ff.) erreicht. ${ }^{7}$

Das Funk-Kolleg Sprache wurde ab Ende September 1971 gesendet. Diese Matrix ist Ergebnis einer längeren Entwicklung. Ein erster Entwurf ist im Beitrag K.-H. Deutrich (1971a: 27f.) veröffentlicht. Sehr anschaulich geht in Abb. 1 das Prototypische der Textsorten aus der Konfiguration der Plus- und Minusmarken hervor.

Der definitorische Prozeß für Textsorte findet in dem Zusammenhang der Eiforschung der Grundstrukturen gesprochener Sprache ihren Abschluß in G. Schank/G. Schoenthal $\left({ }^{2} 1983 /{ }^{1} 1976:\right.$ 4, 5, 40, 42). Es konnten natürlich nur Textsorten im Feld gesprochener Texte erkannt werden. Verlief dieser definitorische Prozeß also in der Annährerung an eine Textsortengliederung der Texte gesprochener deutscher Standardsprache, so kam es alsbald zur Ausweitung auf andere sprachliche Kommunikationsbereiche mit hohem Textaufkommen. Dazu stelle ich als Nachweis eine weitere (1975). 
Matrix vor (Abb. 2), die aufgenommen ist in Kleine Enzyklopädie. Deutsche Sprache (1983: 236).

Abb. 1

(verkleinert)

\begin{tabular}{|c|c|c|c|c|c|c|c|}
\hline \multirow[t]{2}{*}{ Merkmalsmatrix } & \multicolumn{7}{|c|}{ Redekonstellationstypen } \\
\hline & & 1 & II II & III II & IV $\mid V$ & $\mathrm{~V} / \mathrm{V}$ & VI \\
\hline \multirow[t]{2}{*}{ 1. Sprecherzahl } & 1.1 ein Sprecher & + & + & + & & & \\
\hline & 1.2. mehrere Sprecher & & & & $+1+$ & ++ & + \\
\hline \multirow[t]{3}{*}{ 2. Zejtreferenz } & 2.1. zeitlos & + & & & + & & \\
\hline & $\begin{array}{l}\text { 2.2. vorzeitig oder } \\
\text { nachzeitig }\end{array}$ & & + & & & & $(+)$ \\
\hline & 2.3. simultan & & & + & & & \\
\hline \multirow{3}{*}{$\begin{array}{l}\text { 3. Verschränkung Text } \\
\text { soz. Situation } \\
\text { (Situztions- } \\
\text { verschränkung) }\end{array}$} & 3.1. keine & + & + & & + & & + \\
\hline & 3.2. schwach & & & & & & \\
\hline & 3.3. stark & & & + & & + & \\
\hline \multirow[t]{2}{*}{ 4. Rang } & 4.1. gleicher Rang & & & & ++ & + & \\
\hline & 4.2. Unterordng. od. Privil. & + & + & + & & & + \\
\hline \multirow{3}{*}{$\begin{array}{l}\text { 5. Grad der } \\
\text { Vorbereitetheit }\end{array}$} & 5.1. speziell vorbereitet & + & & & $(+)$ & & $(+)$ \\
\hline & 5.2. routiniert & & & & & & \\
\hline & 5.3. nicht vorbereitet & & & & & + & \\
\hline \multirow{3}{*}{$\begin{array}{l}\text { 6. Zahl der } \\
\text { Sprecherwechsel }\end{array}$} & 6.1. null & + & + & + & & & \\
\hline & 6.2. relativ wenig & & & & + & & + \\
\hline & 6.3. relativ viel & & & & & + & \\
\hline \multirow[t]{2}{*}{ 7. Themafixierung } & $\begin{array}{l}\text { 7.1. Thema in voraus } \\
\text { festgelegt }\end{array}$ & + & + & + & + & & $(+)$ \\
\hline & $\begin{array}{l}\text { 7.2. nicht im voraus } \\
\text { festgelegt }\end{array}$ & & & & & + & \\
\hline \multirow[t]{3}{*}{$\begin{array}{l}\text { 8. Modalität der } \\
\text { Themenbehandlung }\end{array}$} & 8.1. deskriptiv & & + & + & & & \\
\hline & 8.2. argumentativ & + & & & + & & $(+)$ \\
\hline & 8.3. assoziativ & & & & & + & \\
\hline \multirow[t]{4}{*}{ 9. Offentlichkeitsgrad } & 9.1. öfentlich & + & & +1 & + & & + \\
\hline & 9.2. halböffentlich & & & & & & \\
\hline & 9.3, nicht öffentlich & & & & & & \\
\hline & 9.4. privat & & $(+)$ & & & + & \\
\hline
\end{tabular}

? Diese Matrix auch in H. STEGER u.a. (1974: 94f.). 
Erläuterungen und Anmerkungen zur Merkmalsmatrix:

Zum Redekonstellationstyp I gehört z. B. die Textsorte "Vortrag"

Zum Redekonstellationstyp II gehören z. B. die Textsorten

Zum Redekonstellationstyp III "Bericht", „Erzählung" gehört 2. B. die Textsorte "Reportage"

Zum Redekonstellationstyp IV gehört 2. B. die Textsorte

Zum Redekonstellationstyp $\mathrm{V}$

Zum Redekonstellationstyp VI "öffentliche Diskussion" gehört z. B. die Textsorte nsmall talk, Unterhaltung" gehört z. B. die Textsorte "Interview*

Die in Anführungszeichen gesetzten Termini - z. B. "Vortrag* sind nicht als alltagssprachliche Ausdrücke zu verstehen, sondern sie sind definiert als sprachstrukturelle Bedingungen der jeweils zu einem Redekonstellationstyp gehörenden Textsorte.

Zur besseren Ubersicht wurde in der Merkmalsmatrix nur das Zeichen + gesetzt, an den Leerstellen ist ein - zu ergänzen.

Die Klammer () zeigt fakultatives Vorliegen einer Merkmalsausprägung an.

Abb. 2

(verkleinert)

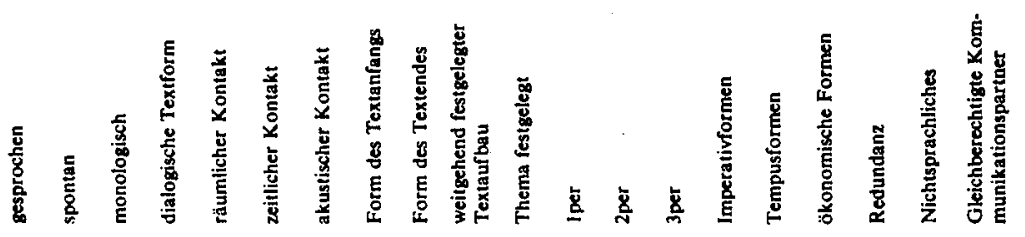

\begin{tabular}{|c|c|c|c|c|c|c|c|c|c|c|c|c|c|c|c|c|c|c|c|c|}
\hline Interview & + & \pm & - & - & \pm & + & + & \pm & \pm & - & + & + & + & + & \pm & \pm & \pm & \pm & + & - \\
\hline Brief & - & \pm & \pm & - & - & - & - & + & + & - & \pm & + & + & + & \pm & \pm & \pm & \pm & + & \pm \\
\hline Telefongespräch & + & \pm & - & - & - & + & + & + & + & - & \pm & + & + & + & \pm & \pm & \pm & \pm & \pm & \pm \\
\hline Gesetzestext & - & - & + & - & - & - & - & + & + & - & + & - & - & + & - & - & - & - & + & - \\
\hline Arztrezept & - & - & + & - & - & - & - & + & + & + & + & - & - & - & - & - & + & - & + & - \\
\hline Kochrezept & \pm & - & + & - & \pm & \pm & \pm & + & - & + & + & - & - & + & \pm & - & \pm & - & + & - \\
\hline Wetterbericht & \pm & - & + & - & - & + & \pm & + & - & + & + & - & - & + & - & - & \pm & - & + & - \\
\hline Traueranzeige & - & - & + & - & - & - & - & + & + & + & + & \pm & - & + & - & - & \pm & - & \pm & \pm \\
\hline Vorlesung(sstunde) & + & \pm & + & - & + & + & + & + & \pm & - & + & \pm & \pm & - & \pm & \pm & - & \pm & \pm & - \\
\hline Vorlesungsmitschrift & - & - & + & - & - & - & - & \pm & - & - & + & - & - & + & - & - & + & - & \pm & + \\
\hline Reklame & \pm & \pm & \pm & \pm & \pm & \pm & \pm & \pm & \pm & - & \pm & \pm & \pm & \pm & \pm & \pm & \pm & \pm & \pm & $\rightarrow$ \\
\hline Stelleninserat & - & - & + & - & - & - & - & + & + & + & + & \pm & \pm & \pm & \pm & - & \pm & - & + & - \\
\hline Rundfunknachrichten & + & - & + & - & - & + & + & + & + & - & - & - & - & + & - & + & - & \pm & + & - \\
\hline Zeitungsnachricht & - & - & + & - & - & - & - & + & - & - & + & - & - & + & - & + & - & - & + & - \\
\hline Telegramm & - & - & + & - & - & - & - & + & + & - & + & \pm & \pm & + & \pm & - & + & - & + & \pm \\
\hline Gebrauchsanweisung & - & - & + & - & - & - & - & \pm & - & - & + & - & \pm & + & \pm & - & \pm & \pm & \pm & - \\
\hline Diskussion & + & \pm & - & - & \pm & + & + & + & + & - & + & + & + & + & \pm & + & - & \pm & + & \pm \\
\hline familiäres Gespräch & + & + & - & \pm & + & + & + & \pm & - & - & - & + & + & + & \pm & \pm & \pm & + & \pm & + \\
\hline
\end{tabular}

Abb. 2.5.-3: Textfypenmatrix (nach SANDIG) 
Sie ist von B. Sandig entworfen in Textsorten (1972). Textklassifikation wurde schon längst in der Didaktik des Schulfaches Deutsch geübt, und zwar im Aufsatzunterricht. Repräsentativ ist dort die Matrix bei H. Helmers ( ${ }^{8} 1975$ : 228f.), die eine Gestaltungslehre für schriftliche und mündliche Schülertexte darstellt.

Auch bei Ausschluß von Einfachen Formen und Gattungen wird man dennoch eine vollständige Erfassung aller Textsorten des übrigen Feldes nicht erreichen können. Dafür ist das Kommunikationsgeschehen zu vielfältig, auch hat man mit einer Metamorphose des Textsorteninventars zu rechnen, in deren Verlauf Textsorten vergehen und neu entstehen. Als ein Metier der Expansion sei z.B. auf das moderne Medium Tagespresse verwiesen; an der dort aufgekommenen Glosse wäre eine solche Textsorteninnovation zu verfolgen.

Im Fachgebiet Textlinguistik bieten moderne Studienbücher selbstverständlich eine Besprechung von Textsorten. Von A. Linke/M. Nussbaumer/P. R. Portmann (21994: 253) wird sogar ein "(intuitives) textsortenspezifisches Textmusterwissen" konstatiert. Es beruhe wahrscheinlich auf der Schaffung von Prototypen von Textsorten, welche die intuitive Zuordnung von Texten zu bestimmten Textsorten ermöglichten. Dort a.a.O., S. 252f. wird die Vielzahl von in die Textlinguistik eingeführten Termini für den Vorschlag einer gestuften Hierarchie genutzt: Texttyp - Textklasse - Textsorte. So wäre der unten besprochene Text 1 ein Textexemplar der Textsorte Glosse, welche dann der Textklasse Kommentar zugeordnet werden könnte; Pressetext wäre die alles umfassencle Textklasse. Die Anzahl der Stufen einer solchen Hierarchie ist relativ, denn man könnte unter dem Hyperonym Gebrauchstext eine weitere Abstraktion anzeigen. Bei B. Imhasly, B. Marfurt, P. Portmann (1979: 170ff.) wird bereits eine entwickelte Textsortenlehre vorgestellt.

In der Gegenüberstellung von Textexemplar und Textsorte werden, wie sonst bei linguistischer Systematisierung, von konkreten Ereignissen der etischen Sphäre die abstraktiven der emischen abgeleitet: Phon - Phonem (Phonetik - Phonemik), Graph Graphem (Graphetik - Graphemik), Morph - Morphem (Morphologie - Morphemik). So könnte man vorschlagen, Textexemplar einer Textetik (Textik?) und Textsorte einer Textemik zuzuordnen. ${ }^{8}$

\section{Exkurs zur Veranschaulichung der Textsorte Glosse}

Da die Textsorte Glosse ins Spiel gebracht worden ist, sollten ihre textlichen Charakteristika aufgezeigt werden. Wie schon gesagt, handelt es sich um eine verhält-

8 Diese Systematik ist ausgeführt bei R. Müller (1993) und könnte um den vorgeschlagenen Schritt zur Textemik-Textetik erweitert werden. Vgl. auch H. Steger u.a. (1974: 46f.) über Textem. 
nismäßig junge Textsorte. Als Textkategorie ist sie noch nicht entschieden identifiziert, obwohl ihre Texte häufig sind und deren Formulierung schon einer ausgeprägten Professionalität bedarf. Das mag daran liegen, daß es sich einerseits um Einfache Formen nicht handelt, daß sie andererseits als journalistische Äußerungen auch noch nicht die Dignität für die Gattungszuordnung haben. Angedeutet wird, daß der Glosse die Funktion des Kommentars zukommt. Die Glosse ist ein kurzer Text, der aktuellen Problemen und Ereignissen in Politik oder Kultur zugewandt ist. Glossen stehen im Zusammenhang journalistischer Texte, denen der Presse, des Rundfunks und des Fernsehens. Sie gelten als feuilletonistisch, insofern sie pointierende Betrachtungen bieten, welche nicht nur im Feuilleton plaziert sind, sondern auch Stellungnahmen in den Bereichen Politik, Wirtschaft, Lokales oder Sport bieten.

Damit etwas Empirie in diese Erörterung gebracht wird, soll hier eine Glosse und ein Bezugstext angeführt werden: 


\section{Text 1}

\section{aus Frankfurter Allgemeine Zeitung \\ (FAZ) Nr. 190, 14.12.1994, S. 35 \\ (verkleinert)}

\section{Polit Oh! logie}

Die Gesellschaft für deutsche Sprache (GfdS) in Wiesbaden sucht, wie auch in dieser Zeitung mitgeteilt wurde, per Ideenwettbewerb einen sinnvollen und passenden Namen für ..männliche Politessen“" Dem maskulinen Pendant zur Knöllchen verteilenden Dame soll nicht länger der Tort (oder vielleicht die Torte?) der Namenlosigkeit oder des umstandskrämerischen Derivativs angetan werden. Wichtig. so die hessischen Sprachpfleger. sei die echte Äquivalenz der Bezeichnung, die auch die Asymmetrie, wie sie etwa zwischen Entbindungshelfer/Hebamme auftrete, zu vermeiden helfe. Aber obwohl wir jetzt seit Tagen bergmäßig kreißen in der Hoffnung, endlich ein Vokalmäuschen zu gebären, von dem wir uns - als Hebammer - natürlich selbst entbinden würden, sind wir dem Ziel um keine Knöllcheniänge näher gekommen. Dabei liegt unser Problem nicht darin, daB uns die linguistische Novität des generischen Femininums, also dieses neuen Adams, der aus Evas Rippe geboren werden soll, sprachlos macht. Es liegt ganz einfach darin, daB die Nachbarparzellen im Schrebergarten des Lautstands allesamt vergeben sind: Polizist kommt nicht in Frage, Politur schafft Glanz, aber keine freien Straßen, Polier gehört der Gewerkschaft Bau, Steine, Erden an. Italienische Auswege wie Policchinello, Poltrone oder Politurione sind uns aus naheliegenden Gründen versperrt. Wäre das Gesuchte sachlicher Natur, kämen Lösungen wie Politüm oder Polistell in Frage. So bleibt zwar noch der Politeur, aber der klingt wie das Gegenstück zu Friseuse, die erst kürzlich wegen schlüpfriger Anklänge aus dem korrekten Vokabular verbann: wurde - von der Masseuse ganz zu schweigen, die seither nur noch in Bahnhofsnähe praktiziert. Und schließlich der Polit, der sehr edel wirkt und sich zum ordinären Polizisten ähnlich distinguiert verhält wie der Anarch zum Anarchisten, aber just wegen so viel kulturellen Adels bald den Neid der Politiker auf sich ziehen würde. Frustriert geben wir auf und warten auf den Helden, der uns der ultimativen Vokaldämmerung entgegenführen wird. Im übrigen ist der Ausweg aus all diesen sprachlichen Sackgassen längst gefunden. Die Berliner, bekannt für ihre linguistische Kompetenz, waren uns wieder einen Zungenschlag voraus. Die Politesse war noch kaum geboren, da hieß sie in Berlin schon die Bulette. Von dieser her rückwirkend das männliche Pendant zu bilden war ein Kinderspiel, zu dem der Volksmund keine drei Sekunden brauchte. Von irgendwelchen akademischen Ehrungen und Preisgeldern für diese Leistung ist uns nichts bekannt.

\section{Text 2}

Aus Frankfurter Allgemeine Zeitung

(FAZ) Nr. 287, 10.12.1994, S. 11

(verkleinert)

\section{Männliche Politesse gesucht \\ Wettbewerb der Sprachgesellschaft}

WIESBADEN, 9. Dezember (AP). Wie lautet die mānnliche Form von Politesse? Einen Ideenwettbewerb zur Lösung dieser kniffeligen Frage hat am Freitag die Gesellschaft für deutsche Sprache (GfdS) in Wiesbaden begonnen. Obwohl das Schreiben von "Knöllchen" inzwischen auch von Männern ertedigt werde, habe man für sie weder in offiziellen Verzeichnissen noch in der Alltagssprache eine Bezeichnung gefunden, sagte Helmut Walther von der GfdS-Zeitschrift „Sprachdienst “.

Zugieich sucht die Sprachgesellschaft auch nach der mānnlichen Entsprechung für die "Postesse“, eine neve Bezcichnung für Postangehörige, die ihr Unternehmen in der Offentlichkeit repräsentieren. "Sie sind schon auf Kongressen und Tagungen aktiv und tragen schöne Postuniformen", sagt Walther. Während die Begriffe Steward und StewardeB ein symmetrisches Wortpaar bildeten, fehlten die männlichen Formen bei der Postesse und der Politesse, offenbar weil diese Berufe überwiegend von Frauen ausgeübt würden. 
Text 1 ist die Glosse. Da er Textsortenexemplar einer Texsorte mit kommentierender Funktion ist, muß man, um seinen Zweck zu durchschauen, auch den kommentierten Text zur Kenntnis geben. Das ist Text 2, in diesem Fall eine Pressemeldung, eine Mitteilung, eine Nachricht. Das Datum erweist den Text 2 als den der Glosse voraufgehenden Anlaß. Im ersten Satz der Glosse wird der Zusammenhang durch ausdrücklichen Verweis hergestellt. In der Zeitung sind die beiden Texte unterschiedlichen Sachgebieten zugeordnet, wobei die Glosse in diesem Fall wegen ihres Themas in das Feuilleton eingebracht ist. Die Glosse erfährt üblicherweise gegenüber der Meldung bzw. den Meldungen eine Hervorhebung, sei es, daß sie durch Rahmung im eigenen "Kasten" erscheint, sei es, daß sie, - wie in diesem Fall -, an einer festen, für Glossen reservierten Stelle erscheint. Die Glosse wird gegenüber der Meldung auch ausgezeichnet, indem sie durch Nennung des Namens oder der Namensinitialen einem Autor zugesprochen wird. Das bewirkt die Unikalisierung des Textes. Die Glosse ist eine persönliche Meinungsäußerung.

Übrigens teilt die Glosse hinsichtlich der Kommentarfunktion die Eigenschaften der nonverbalen Karikatur. Nicht nur, daß sie wie diese auf ein Bezugsereignis bzw. eine Bezugsäußerung gerichtet ist, sie ähnelt ihr darüber hinaus auch in stilistischer Hinsicht, insofern sie satirisch verfährt. Die in der Glosse geübte Kritik wird durchaus polemisierend, ironisierend und spottend vorgetragen. Im vorliegenden Beispiel ist ganz typisch, daß sie dem Inhalt der Meldung vor allem unernste Aspekte abgewinnt. Die Aufforderung zum wortschöpferischen Wettbewerb wird angenommen, dieser dann in ironischer Manier mit Vorschlägen lexikologisch akzeptabler, wahrscheinlich jedoch unerwünschter Analogien übertrieben. Mit dem Angebot eines gar nicht gefragten Resultats wird die Satire auf die Spitze getrieben.

Das Prototypische einer Textsorte hat sich hier manifestiert, so daß man diese Glosse als dieser zugehöriges Textexemplar identifizieren kann. ${ }^{9}$

\section{Natürliche Sprachformen oder kulturelle Sprachformen?}

Im Titel dieses Beitrags steht eine Frage, die aufgrund der bisherigen Feststellungen beantwortet werden soll.

Man kann die Antwort kaum in der Art des "Entweder-Oder" geben, eher im Sinne der Positionsbestimmung in dem semantischen Differential zwischen "natürlich" und "kulturell". "Natürlich" soll hier nicht den Status eines Systems außerhalb der menschlichen Kommunikation bezeichnen, etwa dessen der sogenannten Bienenspra-

In der philologischen Terminologie ist Glosse polysem; vgl. Wilpert, Gero von ( $\left.{ }^{7} 1989\right)$, Metzler-Literatur-Lexikon: Stichwörter zur Weltliteratur (1984). Die hier gemeinte Bedeutung wird mit der Intensivierung der Textlinguistik vorangig werden. Satirische Äußerungen werden didaktisch behandelt von Fritz, Jürgen (1980). 
che. Die gemeinte Qualität von natürlich trifft auf die Texte der Einfachen Formen eigentlich zu, gelten diese doch als naturgegeben, insofern man weder einen individuellen Autor namhaft machen, noch eine initiale Formulierung identifizieren kann. ${ }^{10}$ Die Texte der Gattungen sind artifiziell, vermitteln den Eindruck, einem zeitlichen Geltungsmoment enthoben zu sein. Sie sind Dokumente, als Unikate von Dichterautoren geschaffen. Insofern kann man für sie eigentlich die kulturelle Existenz konstatieren. ${ }^{11}$

Als eher kulturell müssen die Texte der Textsorten gelten, die aktuell und hinsichtlich ihrer zeitlichen Geltung in die gegenwärtige Situation verschränkt sind. Als Produkt von Klassifizierung hat die Textsorte eine begriffliche Realität gewonnen, die Realität eines philologischen Konstrukts. Das deutet auch auf kulturelle Installierung, was die Textsorte sogleich objektiviert zur Anweisung für konkrete Formulierung und als sprachdidaktischen Gegenstand. Dies heißt nicht, daß Textsorten nicht auch real wären im Sinne einer vorwissenschaftlichen Präsenz und mentalen Existenz; das deutet wiederum auf natürliches Vorhandensein.

Zur Erläuterung dieses Paradoxes sei noch einmal auf den Witz verwiesen. Dies geschieht zu dem Zweck, die Metamorphose einer Textsorte von der Existenz als natürlicher Sprachform zu kultureller Sprachform zu beobachten. Der Witz ist eine in dieser Hinsicht gut untersuchte Erscheinung des sprachlichen Handelns. Die Studie B. Marfurts (1977) wurde schon genannt. Auch ohne diese Studie und ohne die Inanspruch-nahme als Einfache Form durch A. Jolles wäre der Witz als spezifische Form sprachlicher Äußerung Realität, sozusagen natürlich. Er zeigt sich als sprachliche Verwirklichung politischer Einstellung, und zwar vom Standpunkt der Beherrschten, als Form von Widerstand oder Affirmation für Vorurteile. ${ }^{12}$ Bei L. Röhrich (1980) ist eine weitergehende Subklassifizierung dieser Textsorte vorgenommen, z.B. ethnischer Witz, Irrenwitz, Professorenwitz etc. Ständiges, alltägliches, und massenweises Auftreten, also eine hohe Textfrequenz, ist dem Witz eigen. In der linguistischen Rezipierung des Witzes als eine Textsorte neben anderen, z.B. auch neben der ähnlichen Anekdote, wird schon seine Einführung unter die kulturellen Sprachformen vollzogen. Die zuvor angezeigte Objektivierung und Didaktisierung hat diese Textsorte auch erfahren. ${ }^{13}$ Von D. Ader (1983) sind Didaktische Überlegungen zum Verständnis der Textsorte "Witz" in dem Zusammenhang Textsorten und Gattungen unter didaktischem Aspekt beigetragen worden. Schon von W. Ulrich (1980) ist eine Didaktik eigens des Witzes in einer Monographie

10 Als Antonym zu "natürlich" ist also mit Absicht nicht "künstlich (unnatürlich)" gesetzt worden; eher, - m.E. jedoch sicherlich nicht treffender -, wäre "künstlerisch" an die Stelle von "kulturell" zu setzen gewesen.

11 Das aufzuzeigen, was die Texte der Gattungen als kulturell erscheinen läßt, ist in R. Müller (1994) versucht.

12 Dafür stehen Titel von Sammlungen wie: H.-J. Gamm (1963); M. Dor/R. Federmann (1964).

13 Bei B. Imhasly/B. Marfurt/P. Portmann (1979:177ff.) wird als beispielhafte Textsorte der Witz präsentiert. 
vorgestellt. Witzrezeption, aber auch -produktion, werden Gegenstand von Lehre, was einen entscheidenden Schritt dahin bedeutet, diese Textsorte (auch von Ulrich so bezeichnet) als kulturell aufzufassen.

Die Antwort hier, Textsorten seien "sowohl" kulturell "als auch" natürlich, scheint ambivalent und wirkt deshalb wenig zufriedenstellend. Das liegt daran, daß man die Prädikate "natürlich" und "kulturell" aus umgangssprachlicher Gewohnheit als oppositionell und das Oder als disjungierend (entweder - oder; lateinisch aut - aut; ausschließendes Oder) versteht. Man kann jedoch auch bedenken, ob hier die Prädikate nicht durch ein adjungierendes Oder (oder auch; lateinisch vel; nicht ausschließendes Oder) verknüpft verstehen soll (W. Kamlah/T. Lorenzen (1967: 145f.)). Es wäre gut, hier das Verhältnis der verknüpften Prädikate so aufzufassen, also im Sinne des adjungierenden Oder.

Die Textsorten sind dann je nach der Perspektive, aus der man sie erlebt bzw. sie sich bewußt macht, natürliche Sprachformen oder kulturelle Sprachformen.

\section{Literatur}

Ader, Dorothea (1983): Didaktische Überlegungen zum Verständnis der Textsorte "Witz". In: Textsorten und Literarische Gattungen. 695-705.

Bausch, Karl-Heinz (1971): Vorschlag zu einer Typik der gesprochenen Sprache. Mit Diskussion zum Vortrag. In: Forschungen zur gesprochenen Sprache und Möglichkeiten ihrer Didaktisierung. 9-39.

Bausinger, Hermann (1980): Formen der Volkspoesie; 2. Auflage. Berlin.

Berens, Franz-Josef (1975): Analyse des Sprachverhaltens im Redekonstellationstyp "Interview". Eine empirische Untersuchung. München.

Bußmann, Hadumod (1990). Lexikon der Sprachwissenschaft. 2. Auflage. Stuttgart.

Deutrich, Karl-Helge (1971a): Aufnahme und Archivierung gesprochener Hochsprache.

In: Texte gesprochener deutscher Standardsprache I. 18-32.

Deutrich, Karl-Helge (1971b): Überlegungen zur Sprechsituation und zum Kommunikationsrahmen. Mit Diskussion zum Vortrag. In: Forschungen zur gesprochenen Sprache und Möglichkeiten ihrer Didaktisierung. 40-70.

Deutrich, Karl-Helge (1973): Redekonstellation und Sprechsituation. Versuch zur Beschreibung eines Kommunikationsaktes. In: Gesprochene Sprache. Bericht der Forschungsstelle Freiburg. 111-192.

Deutrich, Karl-Helge/Schank, Gerd (1973): Redekonstellation und Sprachverhalten I. In: Funkkolleg Sprache. Eine Einführung in die moderne Linguistik. Band II. 242252.

Dor, Milo/Federmann, Reinhard (1964): Der politische Witz. München, Wien, Basel. Elmauer, Ute u. Müller, Rolf (1974): Belegung der Freiburger Forschungshypothese über die Beziehung zwischen Redekonstellation und Textsorte. In: Gesprochene Sprache. Jahrbuch 1972 (1974). 98-120. 
Engel, Ulrich (1969): Das Mannheimer Corpus. In: Forschungsberichte des Instituts für deutsche Sprache 3. 75-84.

Forschungen zur gesprochenen Sprache und Möglichkeiten ihrer Didaktisierung (1971), Protokoll eines Werkstattgespächs des Goethe-Instituts am 10. und 11. Dezember 1970. Hrsg. vom Goethe-Institut, Referat für Unterrichtstechnologie und Mediendidaktik. München.

Forschungsberichte des Instituts für deutsche Sprache 3 (1969), hrsg. von Hugo Moser u.a. (Schriftleitung: Ulrich Engel). Mannheim.

Fritz, Jürgen (1980): Satire und Karikatur. Fächerübergreifender Unterricht in Deutsch - Politik - Kunst - Musik. Braunschweig.

Funk-Kolleg Sprache. Eine Einführung in die moderne Linguistik. Band II (1973). Fischer Taschenbuch 6112. Frankfurt/M.

Gamm, Hans-Jochen (1963): Der Flüsterwitz im Dritten Reich. München.

Gesprochene Sprache. Bericht der Forschungsstelle Freiburg (1973). Forschungs-

berichte des Instituts für deutsche Sprache 7. Hrsg. von U. Engel und I. Vogel.

Mannheim.

Gesprochene Sprache. Jahrbuch 1972 (1974). Hrsg. von Hugo Moser u.a. Düsseldorf. Goppelt, Leonhard (1990, Nachdruck von 1939): Typos. Die typologische Deutung des Alten Testaments im Neuen. Darmstadt.

Helmers, Hermann (1975): Didaktik der deutschen Sprache. 8. Auflage. Stuttgart.

Imhasly, Bernard/Marfurt, Bernhard/Portmann, Paul (1979): Konzepte der Linguistik. Eine Einführung. Wiesbaden.

Jolles, André (1958; Neudruck von 1. Auflage 1930): Einfache Formen. Darmstadt.

Kamlah, Wilhelm/Lorenzen, Paul (1967): Logische Propädeutik oder Vorschule des vernünftigen Redens. Revidierte Ausgabe. Mannheim.

Kern, Peter (1969): Bemerkungen zum Problem der Textklassifikation. In: Forschungsberichte des Instituts für deutsche Sprache 3. 3-23.

Kleine Enzyklopädie. Deutsche Sprache (1983). Hrsg. von Joachim Schildt u.a. Leipzig.

Kreuder, Hans-Dieter (1993): Studienbibliographie Linguistik. 3. Auflage. Stuttgart.

Kürschner, Wilfried (1994): Taschenbuch Linguistik. Ein Studienbegleiter für Germanisten. Berlin.

Linke, Angelika/Nussbaumer, Markus/Portmann, Paul R. (1994): Studienbuch Linguistik. 2. Auflage. Tübingen.

Marfurt, Bernhard (1977): Textsorte Witz. Möglichkeiten einer sprachwissenschaftlichen Textsorten-Bestimmung. Tübingen.

Metzler-Literatur-Lexikon: Stichwörter zur Weltliteratur (1984). Hrsg. von Günther und Irmgard Schweikle: Stuttgart.

Müller, Rolf (1973): Die Konzeption des Corpus gesprochener Texte des Deutschen in der Forschungsstelle Freiburg des Instituts für deutsche Sprache. In: Gesprochene Sprache. Bericht der Forschungsstelle Freiburg. 47-75.

Müller, Rolf (1993): "Etisch" und "Emisch" in der Semantik. In: Wortschatz - Satz Text. 249-255. 
Müller, Rolf (1994): Das Poetische an einem literarischen Text. Expliziert an Johannes Bobrowski, Stiller Sommer; zugleich etwas über Wachteln. In: Texttyp, Sprechergruppe, Kommunikationsbereich. Hrsg. von Heinrich Löffler, Karlheinz Jakob und Bernhard Kelle. 150-165.

Müller, Werner (1969): Teilerhebungen und ihre Anwendung auf die Sprachbearbeitung. In: Forschungsberichte des Instituts für deutsche Sprache 3. 55-74.

Probleme des Deutschen als Fremdsprache. Forschungsberichte und Diskussionen.

Bericht von der 1. Internationalen Deutschlehrertagung 1967 in München. Hrsg. und eingeleitet von Manfred Triesch. München.

Röhrich, Lutz (1977): Der Witz. Seine Formen und Funktionen. Mit tausend Beispielen in Wort und Bild. München.

Sandig, Barbara (1972): Zur Differenzierung gebrauchssprachlicher Textsorten im Deutschen. In: Textsorten. Differenzierungskriterien aus linguistischer Sicht. Hrsg. von E. Gülich und W. Raible. Frankfurt. 113-124.

Satz und Wort im heutigen Deutsch (1967). Probleme und Ergebnisse neuerer Forschung. Jahrbuch 1965/1966 des Instituts für deutsche Sprache. Hrsg. von Hugo Moser u.a. Düsseldorf.

Schank, Gerd/Schoenthal, Gisela (1983): Gesprochene Sprache. Eine Einführung in Forschungsansätze und Analysemethoden. 2. Auflage. Tübingen.

Schröder, Peter (1973): Die Untersuchung gesprochener Sprache im Projekt "Grundstrukturen der deutschen Sprache". Planungen - Probleme - Durchführung. In:

Gesprochene Sprache. Bericht der Forschungsstelle Freiburg. 5-46.

Steger, Hugo (1967): Gesprochene Sprache. Zu ihrer Typik und Terminologie. In: Satz und Wort im heutigen Deutsch. 259-291.

Steger, Hugo (1969): Forschungsbericht: Gesprochene Sprache. In: Probleme des Deutschen als Fremdsprache. 80-99.

Steger, Hugo u.a. (1974): Redekonstellation, Redekonstellationstyp, Textexemplar, Textsorte im Rahmen eines Sprachverhaltensmodells. Begründung einer Forschungshypothese. In: Gesprochene Sprache. Jahrbuch 1972 (1974). 39-97.

Steger, Hugo (1983): Über Textsorten und andere Textklassen. In: Textsorten und literarische Gattungen. 25-67.

Texte gesprochener deutscher Standardsprache I (1971). Erarbeitet im Institut für deutsche Sprache. Forschungsstelle Freiburg i. Br. München.

Textlinguistik (1978). Hrsg. von Wolfgang Dressler. Darmstadt.

Textsorten. Differenzierungskriterien aus linguistischer Sicht (1972). Hrsg. von E. Gülich und W. Raible. Frankfurt/M.

Textsorten und Literarische Gattungen (1983). Dokumentation d. Germanistentages in Hamburg vom 1..4. April 1979. Hrsg. vom Vorstand d. Vereinigung d. Dt. Hochschulgermanisten. Berlin.

Textsortenlehre - Gattungsgeschichte (1977). Hrsg. von Walter Hinck. Heidelberg. Texttyp, Sprechergruppe, Kommunikationsbereich. Studien zur deutschen Sprache in Geschichte und Gegenwart (1994). Hrsg. von Heinrich Löffler, Karlheinz Jakob u. 

York.

Ulrich, Winfried (1980): Der Witz im Deutschunterricht. Braunschweig. Werlich, Egon (1979): Typologie der Texte. Entwurf eines textlinguistischen Modells zur Grundlegung einer Textgrammatik. 2. Auflage. Heidelberg.

Wilpert, Gero von (1989): Sachwörterbuch der Literatur. 7. Auflage. Stuttgart.

Wortschatz - Satz - Text. Beiträge der Konferenzen in Greifswald und Neubrandenburg 1992 (1993). Hrsg. von G. Bartels und I. Pohl. Frankfurt/M., Berlin, Bern u.a. Zimmermann, Heinz (1965): Zu einer Typologie des spontanen Gespräches. Syntaktische Studien zur baseldeutschen Umgangssprache. Bern.

\section{Zusammenfassung}

Der Beitrag unter diesem Titel soll zu einer Bestandsaufnahme der inzwischen arrivierten Textlinguistik beitragen. Texte als sprachliche Erscheinungsformen haben schon immer existiert. Sie sind auch immer bewußt gewesen, was insbesondere für die Literaturwissenschaft mit ihrem Engagement für poetische Texte gilt. Es können zumindest die Texte im vorliterarischen und nichtpoetischen Feld der Erfahrung als natürlich gelten. In den letzten drei Jahrzehnten ist nach intensiver Hinwendung zur Syntax als der Wissenschaft vom Satz, zur Semantik und zu einigen anderen Anwendungsgebieten der Text besonders interessant für die Sprachwissenschaft geworden. Das hat zur Etablierung einer Textlinguistik geführt. Als deren hauptsächlicher Ertrag zeigt sich die Identifizierung der Textsorten. Die Textsorten haben zwar auch schon wie die Einzeltexte immer existiert, erscheinen jedoch weniger natürlich, da sie insbesondere durch wissenschaftliche Reflexion bewußt gemacht sowie eher abstrakt und begrifflich präsent(iert) sind. Das Prototypische ähnlicher Texte wird als Textsorte begriffen. Mit Absicht ist im semantischen Differential nicht die Qualifizierung künstlich (unnatürlich) als antonymisch zu natürlich. gesetzt worden, sondern kulturell. Als Konstrukte wissenscahftlicher Reflexion sind Textsorten kulturelle Sprachformen, was sich daran zeigen läßt, daß sie in diesem Prozeß der Erhebung von der unwillkürlichen Existenz zu willkürlicher Ver- oder Anwendung gelangen. An der Textsorte Glosse in den Medien Presse und Funk läßt sich dieser Zusammenhang veranschaulichen. Im Beitrag soll dieser Vorgang der Identifizierung der Textsorte wissenschaftsgeschichtlich aus der Perspektive der germanistischen Sprachwissenschaft verfolgt werden.

Povzetek

BESEDILNE VRSTE - NARAVNE ALI KULTURNE JEZIKOVNE OBLIKE?

Članek s tem naslovom naj bi ugotovil, do katerih spoznanj je do sedaj prišlo besediloslovje. Besedila kot jezikovne pojavne oblike so vedno obstajala. Prav tako so se jih vedno zavedali, kar še posebej velja za literarne vede $z$ njihovim angažmajem za poetična besedila. Za naravna lahko veljajo vsaj besedila $v$ predliterarnem in nepoetičnem območju izkušenj. V zadnjih treh desetletjih je po intenzivni usmeritvi $\mathrm{k}$ stavku, semantiki in nekaterim drugim uporabnim področjem postalo besedilo še posebej zanimivo za jezikoslovje. To je pripeljalo do uveljavitve besediloslovja. Kot njegov bistveni rezultat se kaže prepoznavanje besedilnih vrst. Besedilne vrste so tako kot posamezna besedila sicer tudi že vedno obstajale, vendar pa se pojavljajo manj naravno, saj jih uzaveščamo predvsem $z$ znanstveno refleksijo ter predstavljamo abstraktno in pojmovno. Prototipičnost podobnih besedil se pojmuje kot besedilna vrsta. Namenoma ni pri pomenskem razlikovanju uporabljena oznaka umetno (nenaravno) kot protipomenka izrazu naravno, ampak kulturno. Kot konstrukti znanstvene refleksije so besedilne vrste kulturne jezikovne oblike, kar se kaže v tem, da preidejo v procesu opustitve spontanega obstajanja k zavestni uporabi. To povezanost je mogoče razložti $z$ besedilno vrsto glosa $v$ tisku in radiu. Članek poskuša opisati proces prepoznavanja besedilne vrste znanstveno zgodovinsko s stališc̆a germanističnega jezikoslovja. 